\title{
Proximal end of 15-year-old ventricular electrode penetrating pulmonary tissue - a source of infection and a challenge for transvenous lead extraction
}

\author{
Maciej Polewczyk ${ }^{1}$, Aneta M. Polewczyk ${ }^{1}$, Andrzej Kutarski ${ }^{2}$, Anna Polewczyk ${ }^{3}$ \\ ${ }^{1}$ District Hospital, Kielce, Poland \\ ${ }^{2}$ Department of Cardiology, Medical University of Lublin, Lublin, Poland \\ ${ }^{3} 2^{\text {nd }}$ Department of Cardiology, Świętokrzyskie Cardiology Centre, Kielce, Poland
}

Postep Kardiol Inter 2015; 11, 3 (41): 248-249

DOI: $10.5114 /$ pwki.2015.54026

We report a case of a 56-year-old man with a DDD pacemaker implanted in 1998 followed by additional ventricular lead implantation in 2000 due to lead dysfunction and battery replacement in 2006 (proximal end of the abandoned lead already observed in the pulmonary artery), with currently present clinical signs of lead-dependent infective endocarditis (LDIE). In March 2013 he was diagnosed with recurrent episodes of shortness of breath, cough and fever. Initially the patient was hospitalized in the pneumology ward and underwent a series of diagnostic examinations, including computed tomography (CT) and bronchoscopy. Chest CT revealed localized signs of inflammation (which could be differentiated from neoplastic lesions) in the mid-right pulmonary lobe. Given the vicinity of the migrated lead to the lung inflammatory process, penetration of the lead in the lung tissue was confirmed. Blood cultures were positive for Staphylococcus epidermidis (MSS). After cardiologist consultation the patient was transferred to the Cardiology Clinic with LDIE diagnosed. The patient was qualified for a transvenous lead extraction (TLE) procedure. Pre-operative transthoracic echocardiography (TTE) revealed dilatation of the right atrium and ventricle with very high pulmonary artery systolic pressure (PASP = $80 \mathrm{~mm} \mathrm{Hg}$ ) calculated from tricuspid regurgitation. There were no signs of vegetations in transesophageal echocardiography (TEE) examination. The TLE procedure was performed in the cardiovascular operating room with on-site surgical standby. General anesthesia was used; invasive blood pressure, ECG, and ventilation parameters were monitored. At the beginning both functional leads were removed (using a Byrd dilator mechanical sheath).
After unsuccessful grasping of the non-functional lead at the level of the right ventricle (due to adherences to the wall), it was successfully captured in the pulmonary artery using a pig-tail catheter; afterwards the proximal end was grasped in the right atrium lumen with a lasso catheter and the lead was finally extracted using the left subclavian approach and dilator sheaths (Figure 1). There were no procedural or post-procedural complications. Full radiological and clinical success was achieved. Postoperative TTE/TEE showed no signs of lead fragments or vegetations and reduction of PASP $(60 \mathrm{~mm} \mathrm{Hg})$. As the patient was not pace-dependent, system reimplantation was delayed.

According to the current guidelines, indications for TLE of a non-functional lead present in the pulmonary bed should be considered as class IIb (extraction during procedure on cardiac implantable electronic device if contraindications are absent, the lead position might become dangerous) or even class Ila (potentially dangerous lead, which does not immediately threaten the patient) but not class III (despite anomalous lead displacement) [1]. Although the TLE complication rate remains low (about 2\%) [2], longer dwelling time of the lead is a known risk factor of TLE, lead adherence to the vessel wall is much stronger after years, which often necessitates the use of extra tools [3]. Moreover, the proximal end of the broken lead in pulmonary tissue might have an impact on development of infection. Having in mind the fact that in spite of technical difficulties, the clinical success rate of TLE in patients with broken, migrating leads is comparable to the "standard" procedure [4], earlier extraction could prevent lead-related infections and reduce the potential procedural risk. 

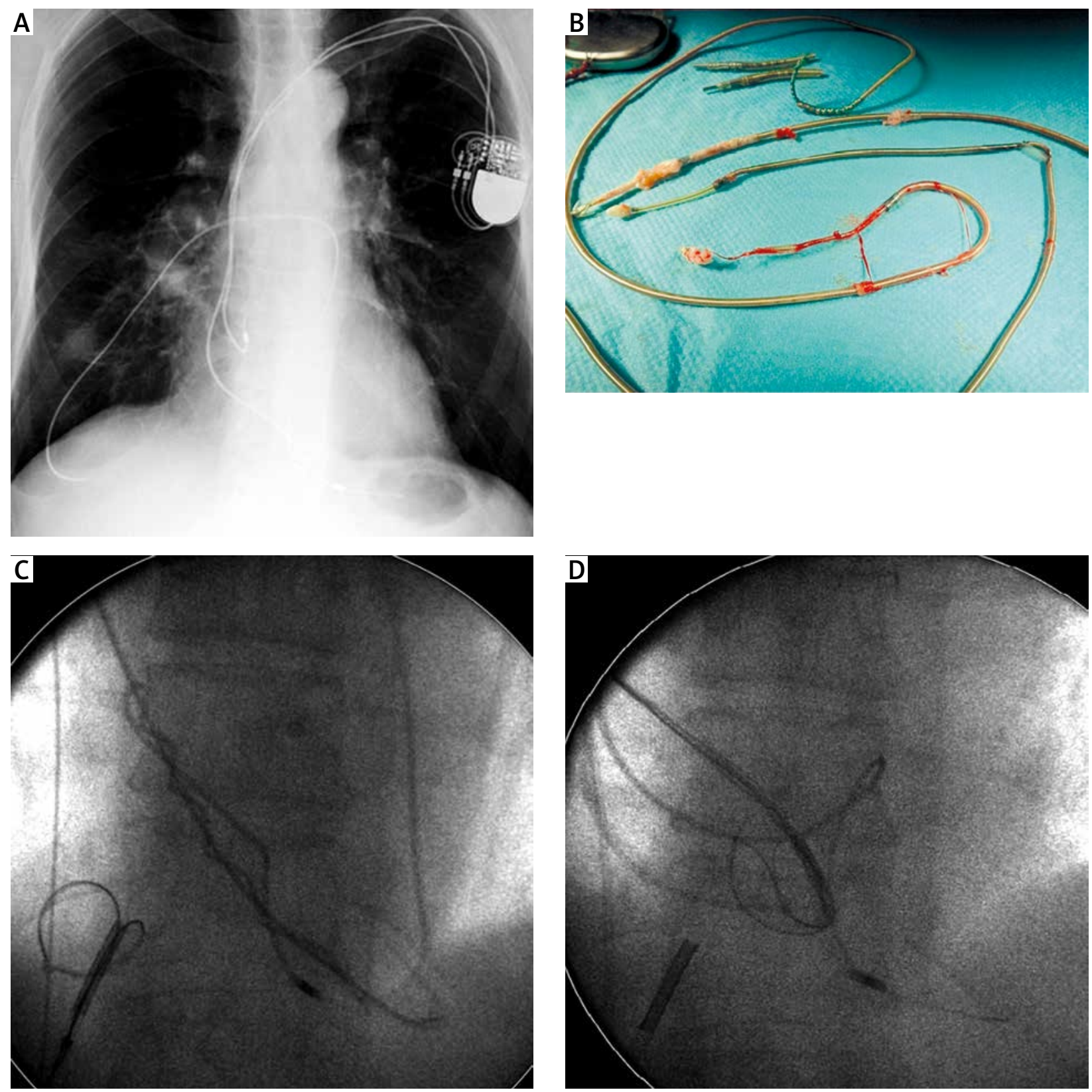

Figure 1. A - Proximal end of the abandoned non-functional lead penetrating pulmonary tissue. B - Removed leads covered with fibrotic tissue. C, D - The lead recaptured with a lasso via the left subclavian approach

\section{Conflict of interest}

The authors declare no conflict of interest.

\section{References}

1. Wilkoff BL, Love CJ, Byrd CL et al. Transvenous lead extraction: Heart Rhythm Society Expert Consensus on facilities, training, indications, and patient management. Heart Rhythm 2009; 6: 1085-104.

2. Wazni O, Epstein L, Carrillo RG, et al. Lead extraction in the contemporary setting: the LExICon Study an observational retrospective study of consecutive laser lead extractions. J Am Coll Cardiol 2010; 55: 579-86.
3. Kutarski A, Pietura R, Czajkowski M. Difficult dual stage transcutaneous lead extraction complicated by fracture of both leads. Cardiol J 2012; 19: 412-7.

4. Kutarski A, Malecka B, Zabek A, et al. Broken leads with proximal endings in the cardiovascular system: serious consequences and extraction difficulties. Cardiol J 2013; 20: 161-9. 